\title{
Utilization of ATR-FTIR Spectroscopy- Multivariate Chemometric Analysis in the Examine of Food Quality Edible Sesame Oils
}

\author{
Selvaraj K, Sankarganesh A, Sajan Francis P, Suraj B
}

\begin{abstract}
Attenuated total reflectance (ATR)-Fourier transform infrared spectroscopy (FTIR) and multivariate chemometric analysis was established to distinguish and classify edible sesame oils of various branded (refined) and unbranded (cold pressed) oils and principal components. For this determination, available branded and unbranded traditional cold pressed sesame oils from southern districts of Tamilnadu, India were qualitative and quantitatively analyzed and authenticated. The FTIR spectra were generated in absorption ranges from 599$4000 \mathrm{~cm}^{-1}$ observed through ATR mode. The generated spectral data combined with multivariate chemometric analyses such as Principal components analysis (PCA), Principal coordinate analysis (PCoA), Agglomerative hierarchical clustering analysis $(A H C)$, Discriminant analysis (DA) were analyzed. Further, observed qualitative analyses through colour, UV-light absorption, absolute viscosity, saponification and iodine values were well differentiated between branded and unbranded sesame oil samples. Based on the multivariate chemometric analysis, unbranded 2 and branded 1 sesame oil samples have shown essential phytocomponents like sesamin, sesaminol, and sesamolin. However, other oil samples did not show those essential phytocomponents and may be missing/eliminated during the refining/filtration processes. In addition, branded and unbranded sesame oil samples have been classified through discriminant analysis (DA) based on the major absorption frequencies. As per the experimental and statistical outcomes, it might be established that ATR-FTIR method has potential to detect and identify essential components of similar food products for classification and authentication, further; it is nondestructive, effective and simple alternative method.
\end{abstract}

Keywords: Chemometric analysis, principal components sesame oil, sesamin, sesaminol

\section{INTRODUCTION}

Sesame seed (Sesamum indicum L.) is high oil yielding dicot crop preferred worldwide among major oil crops with its top most cultivation in tropical and subtropical regions of Asian and African countries ${ }^{1}$. Its composition includes 44$58 \%$ oil, $18-25 \%$ protein, $\sim 13 \%$ carbohydrates. Sesame seed is consumed in its raw or edible oil extracted from it and even its seed cake is traditionally used as cattle feed. Presence of few anti-oxidative phytochemicals, lignants, phenolic compounds, flavonoids, etc., ${ }^{2}$ makes sesame a

Revised Manuscript Received on December 05, 2019.

K. Selvaraj, Assistant Professor, Department of Biotechnology, Kalasalingam University, Tamilnadu.

A.Sankar Ganesh, Department of Biotechnology, Kalasalingam Universityof Research and Education, Tamilnadu.

P Sajan Francis, Pursuing Bachelor of Pharmacy, Karavali college of pharmacy, NH 169, vamanjoor, Mangalore-575028.

B. Suraj, Department of Biotechnology, , Kalasalingam Universityof Research and Education, Tamilnadu. healthier diet and hence research interests focuses on exploration of other beneficiary compounds. Sesamin, sesamolin, sesaminol are lignants present in sesame possessing antitumor and health promoting activities have been one of the major study ${ }^{3}$. Sesame oil play essential role in diet, it is very commonly used in South Indian cuisines. It has also been used as a flavour enhancer in Chinese, Korean and certain other Southeast Asian cuisines ${ }^{4}$. Sesame oil has high proportion of $41 \%$ polyunsaturated fatty acid (Omega6-fatty acids/linoleic acid), 39\% of monounsaturated fatty acids (Omega-9-fatty acids/Oleic acid), 13\% saturated fatty acids (Palmitic acid, Stearic acid), and traces of protein, vitamins, carbohydrates, etc., ${ }^{5,6}$ Due to higher contents of polyunsaturated and monounsaturated fatty acids, it is healthier to use them than oils with higher saturated fatty acids levels ${ }^{7}$. Moreover, polyunsaturated fatty acids, said to improve regulating blood cholesterol level ${ }^{8,9}$. In general, there are many variations in sesame oils due to processing and refining for example Cold-pressed sesame oil is mostly colorless, while mostly all Indian refined sesame oils (gingelly or til oil) are golden in color and Chinese sesame oils are commonly dark brown colour ${ }^{10}$. This dark color and flavour are obtained as of roasted/toasted sesame seeds. Similarly, cold-pressed sesame oil has less flavour than the toasted oil, since it is produced directly from raw, rather than toasted seeds. Sesame is a major edible oil contributor in many parts of the world. Its seeds are segregated qualitatively and high grade seeds are meant for oil production. At present industries under food sector has shown tremendous growth with respect to the increase in demand. This sudden acceleration in need for product and its production in a limited time period has overburdened the availability of raw materials which leads to adulteration of products with compounds sharing similar chemical, physical characteristics which would get miscible with the end product. Food sector has become a major contributor of adulteration to meet the increasing needs of outnumbered population despite of numerous laws, boards regulated by governments for food safety and security.

As food safety and quality that are viewed important issues are specifically identified with the human wellbeing and social advancement individuals progressively search for trusted brands of food products, and anticipate that manufacturers and retailers will give top notch items. Oil purification is one of the major practices all over the industrial sectors for increased consumer attraction and commercial value. Some common consumer attractive practices in oil processing may include filtration, repeated heating, additives like coloratives, miscible oils, and fragrances to increase the oils qualitative and quantitative parameters at low production cost. Among these steps

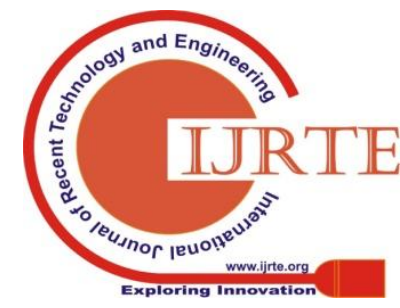


prolonged thermal exposure of sesame oil leads to cleavage of sesamolin, polyunsaturated fatty acids and lignants essential for human health present in raw sesame oil ${ }^{11,12}$. These practices may severely affect the micro and macro nutraceuticals of sesame combined with generation of various health issues to the consumer. NMR, PCR, ESI-MS fingerprinting analysis, etc., are used to identify the adulteration and the essential principal components of edible oils. Though, few of these analytical techniques are time consuming, required specialized procedures for analysis but also destructive methods. Therefore, alternative analytical method which is rapid and easy to operate also a

popular instrumental technique requires very little sample and less time consuming and non-destructive is essential. In this scenario, ATR-FTIR analytical techniques along with multivariate chemometric analysis were used to detect and statistically identify the principal components present in the edible sesame oil samples. FTIR spectroscopy is one of the most important and developing tool used for research related to food materials on the basis of functional groups for over 15 years. This technique is fast and sensitive with great variety of sampling techniques. It is well established

\section{EXPERIMENTAL SECTION}

\section{A. Materials}

Edible sesame oils including 9 brands of commercial oils from supermarkets and 2 unbranded oils from local producer were acquired from southern districts of Tamilnadu, India. The selected oil samples were kept in the dark place at room temperature until the day of analysis. Before analysis, the color and physical nature of oil samples was observed through naked eye, absolute viscosity by Ubbelohde capillary type viscometer, UV-visible and ATRFTIR spectrophotometer.

\section{B. Physical and chemical properties attainment}

Each oil samples were placed in an ultra-clear glass test tube $(\sim 5 \mathrm{~mL})$ and observe the color of oil samples under white background. The UV visible absorption spectrum of each oil samples were carried out by Shimadzu (UV-1800 series, UV Probe 2.62 software, Japan) UV visible spectrophotometer in the range of $200-550 \mathrm{~nm}$ at $0.5 \mathrm{~nm}$ interval, approximately 5 min for complete data collection time. The chemical properties of saponification and iodine value of edible oils indicates the degree of in saturation, and measure the total fatty acids bonded or not, respectively. The saponification and iodine value analyses of each sesamD. oil samples were performed according to AOAC, 1993 and ISO 3657,2013 standards $^{18,19}$. Further, the absolute viscosity of the oil samples was determined at $28^{\circ} \mathrm{C}$ in an automatic system of Ubbelohde capillary type viscometer, with $30 \mathrm{~cm}$ length and $0.05 \mathrm{~cm}$ inner radius. Approximately, $25 \mathrm{~mL}$ of oil samples was placed in a tube and measure the time of flowing using stop watch. The dynamic or absolute viscosity $(\mu)$ and kinematic viscosity (v) can be interlinked by $\mathrm{v}=\mu / \rho$

Where, $\mathrm{v}=$ kinematic viscosity (stoke $(\mathrm{St})$ or centistoke (cSt), $\mu=$ absolute or dynamic viscosity $\left(\mathrm{Ns} / \mathrm{m}^{2}\right.$ or $\mathrm{Pa} . \mathrm{S}$ or $\mathrm{Kg} / \mathrm{m} . \mathrm{s}), \rho=$ density $\left(\mathrm{kg} / \mathrm{m}^{3}\right)$

\section{FTIR spectra attainment}

Different analytical techniques such as HPLC, GC-MS,

analytical technique in the analysis of edible oils and fats ${ }^{13}$. Further, it is a non-destructive technique and needs minimum sample preparation with attenuated total reflection (ATR) apparatus. Many research works have been published to detect and identification of adulteration in various edible oils $^{14-17}$. The present study examined ATR-FTIR technique as a reliable method to detect and identify the principal components present in the sesame edible vegetable oils available in the southern districts of Tamil Nadu, India as branded and unbranded oil samples were obtained from the stores off the self for authentication and classification. Presently, the development of computer science in software technology and statistical multivariate chemometric analysis has been widely used along with FTIR spectroscopy for quantitative analysis. In addition, qualitative analyses of color, light absorption, absolute viscosity, saponification value and iodine values were to be useful for monitoring the adulterations and purity of valuable edible oils. In this research, we have used ATR-FTIR spectroscopic method combined multivariate chemometric analysis to distinguish phytocomponents such as sesamin, sesaminol, sesamolin through principal components, principal coordinate, and discriminant analysis to distinguish and classify the various branded ( 9 samples) and unbranded (2 samples) sesame oils.

ATR-FTIR analysis of sesame oil samples were carried out by a pipetting a small drop $(\sim 5 \mu \mathrm{L})$ of oil covered on the surface in contact with ATR sample baseplate at a controlled room temperature $\left(30^{\circ} \mathrm{C}\right)$. The ATR-FTIR spectral data were generated on a Shimadzu IR Tracer-100 equipped with a sample placement on single-reflection "Golden Gate" diamond ATR module with dimensions of $10 \times 60 \mathrm{~mm}$, producing 12 internal reflections with a penetration depth (infrared beam) of $2.0 \mu \mathrm{m}$, composed of zinc selenide (ZnSe) crystal having refractive index of 2.4 at $1000 \mathrm{~cm}^{-1}$ and operated in absorption range of $599-4000 \mathrm{~cm}^{-1}$ at a resolution of $4 \mathrm{~cm}^{-1}$, approximately $2 \mathrm{~min}$ for complete data collection time. The equipment is attached with advanced library search functions include spectral search, text search, compound search, and peak search incorporating Shimadzu's unique search algorithms for a high hit rate connected to computer and operated by Labsolution IR software, Shimadzu, Japan. The plate was carefully cleaned twice by hexane followed by acetone and dried with soft tissue before filling in with next sample. A background was collected before each oil sample was analyzed and then deducted automatically from the sample spectra prior to next analysis.

\section{Statistical analyses}

ATR-FTIR generated spectral data were compressed and performed the statistical techniques of multivariate chemometric analysis using XLSTAT software (version 2015.4.01.22368, Addinsoft, New York, NY, USA). Graphing was carried out by OriginPro software (version 2015 (64 bit) SR1 b9.5.1.195, OriginLab Corporation, Northampton, MA 01060 USA). The multivariate chemometric analysis was established by preprocessing method of Savitzky-Golay (SG) algorithm with second order polynomial and 11 smoothing points. Later, data were mean-centered and standardized using the standard normal variate transformation. The principal components analysis (PCA), Principal Coordinate analysis $\quad(\mathrm{PCOA}), \quad$ and

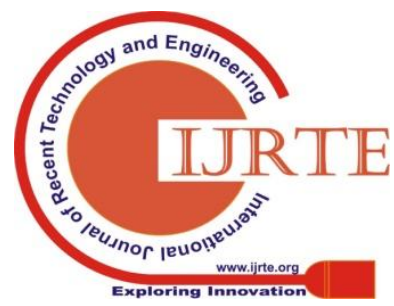


agglomerative hierarchical cluster analysis (AHC) analyses were used for the authenticity and identification of essential components of sesame oils, and discriminant analysis (DA) for their further discrimination. A statistical procedure based on PCA analysis was utilized to emphasize the differences between sesame oil samples. PCA uses on orthogonal transformation into a set of potentially large number of correlated variables into a set of values of linearly uncorrelated variables, and thus reduce the size of the data set. PCA permitted the identification of the most important variables (wavenumbers) associated with the differences between oil samples. PCoA attempts to represent the distance between branded and unbranded sesame oil samples. In particular, it maximizes the linear correlation between the distances in the distance matrix, and the distances in a space of low dimension. AHC was executed to group similar oil samples through a dendrogram construction. The chosen distance to estimate the variety dissimilarity component was Euclidean and Ward's method was used for agglomerative hierarchical clusteringB. Eradication of uncommunicative spectral variables is important to achieve more robust and less complex models, nevertheless able to predict sesame oil quantification.

\section{RESULT AND DISCUSSIONS}

\section{A. Physical and chemical properties attainment}

Sesame oil samples showed minor variation in color when observed under white light background. The color difference of the oil samples was displayed in Table 1. The UV absorption spectra of branded and unbranded sesame oil samples are shown in Figure 1. They demonstrated minor differences in the absorption intensity and peak ( $\lambda \max )$, especially in the wavelength of 348-354 nm. The absorption peak $(\lambda \max )$ of branded oil sample 1,3 displayed $350 \mathrm{~nm}$, branded oil samples 2, 4, 6, 7, 8 and 9 displayed $352 \mathrm{~nm}$, branded oil sample $348 \mathrm{~nm}$ and unbranded oil sample 1 and 2 showing $354 \mathrm{~nm}$. The absorption intensity of branded oil sample 5 had lowest absorption peak ( $\lambda$ max). While, others (both branded and unbranded oil samples) were showing highest intensity. These differences demonstrated and distinguished the branded and unbranded sesame oils. All branded oils showing lower absorption peak intensities as compared to unbranded oils, the observed results have shown that branded oils are highly refined and minute amounts of decolorizing agents used probably revealing clear color of the oil. Further, the results of absolute viscosity have shown extensive variation in branded and unbranded oils, and the range of variability displayed is shown in Table 1. The reason behind the lower absolute viscosity of branded oils might be due highly refined processing, or the addition of decolorizing agents/removal of uncrushed/unfiltered fine particles (sesame seed) present in the oil. The changes of physical and chemical properties of sesame oil can be used to identify the sensory and nutritional quality of food. Viscosity of oil is responsible for the hardness and softness of food, whereas, saponification and iodine values are indicative of the essential components of fatty acids.

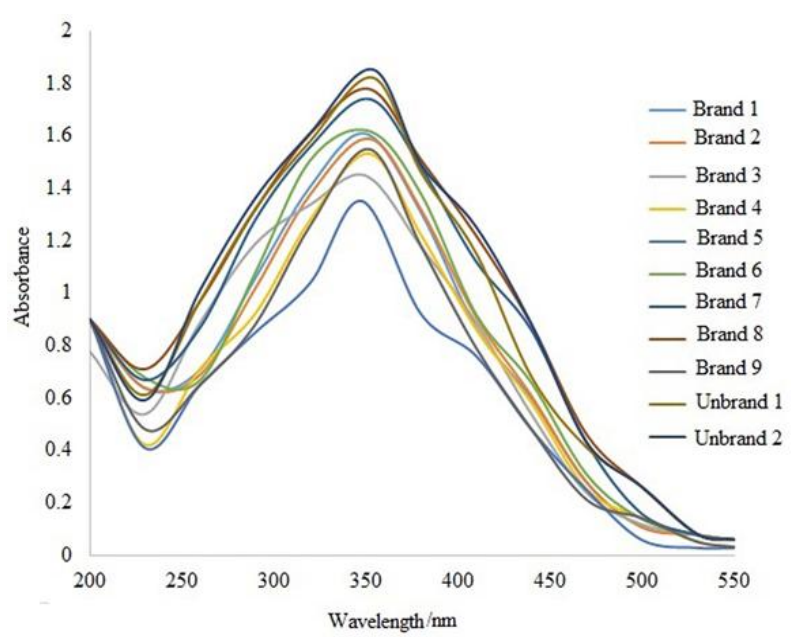

Fig. 1: The UV absorption spectrum of branded (1-9) and unbranded $(1,2)$ sesame oil samples

\section{Qualitative analysis of FTIR}

In this work, various branded and unbranded sesame oils were characterized using ATR-FTIR has shown that the identification of essential lignants and classification of sesame oils of various trademarks (branded or unbranded). Fig. 2 show the FTIR absorption spectra of sesame seed oil (brand 1 and unbrand 2) in the $599-4000 \mathrm{~cm}^{-1}$, the major characteristic peaks of spectra were listed in Table 2. Further, ATR-FTIR spectrum of sesame oil samples were collected in mid IR region $\left(599-4000 \mathrm{~cm}^{-1}\right)$ has been used to identify functional groups and the bands corresponding to vibration in the selected sesame oil samples (Fig. 3(a)). All spectra have shown fairly similar looking peaks and it was difficult to identify the principal components/adulterants because of all available vegetable oils essentially composed of highest contents of triacylglycerol (92\%), 5\% of di- and monoacylglycerols and small levels of other components. However, there was minor changes between two spectra (branded 1 and unbranded 2 oils) which can be noted through the wavenumbers of $3416 \mathrm{~cm}^{-1}$ caused by $-\mathrm{OH}$ group of Sesamolin, sesamin and sesaminol, $2354 \mathrm{~cm}^{-1}$ assigned $-\mathrm{C}=\mathrm{O}$ group of unsaturated fatty acids at $854 \mathrm{~cm}^{-1}$ suggested - $\mathrm{CH}$ of two adjacent hydrogens at 3,4 positions. Sesaminol, sesamin and sesaminol are to be present in trace amounts in sesame oil as a phenolic antioxidant factors. The mentioned phenolic antioxidant factors and other traced functional elements might have been eliminated/liberated during the process of refining in the case of branded oil samples. Further, the fingerprint regions $\left(800-1200 \mathrm{~cm}^{-1}\right)$ of FTIR spectral data were divided in two parts like as 800-900 $\mathrm{cm}^{-1}$ and 900-1200 $\mathrm{cm}^{-1}$ (inset Fig. 3(b) and 3(b) respectively) and there are minor differences between the oil samples were observed, distinguishing the oils of different brands or trademarks showing that the changes of unsaturated to saturated fatty acid due to repeated refining process/variations in processing/quality control practices. 


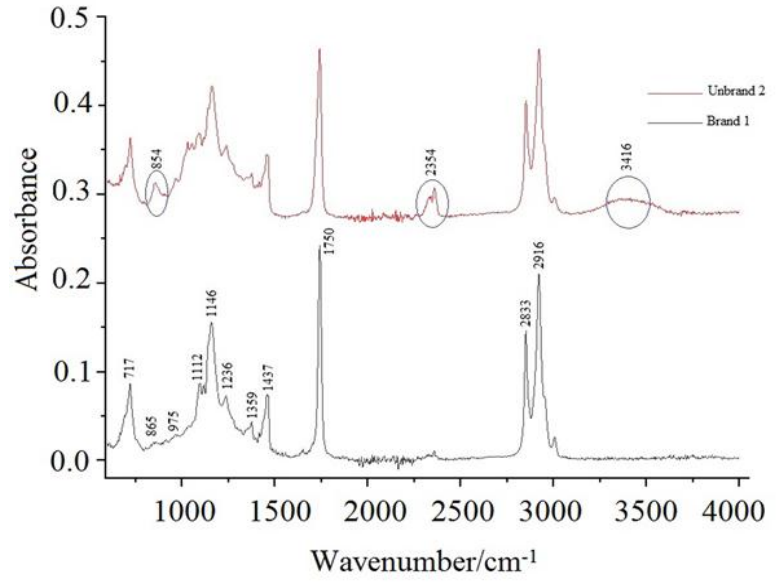

Fig. 2: FTIR absorption spectra of brand 1 and unbranded 2 sesame seed oil

\section{Principal component analysis (PCA)}

PCA is a statistical technique within the context of multivariate analysis. PCA is a well-established technique that reduces the data dimensionality by performing a covariance analysis between factors ${ }^{20,21}$. It was performed to expose the most significant characters in the dataset and the similarities between sesame oil samples. Fig.4 (a) and (b) illustrated the PCA score plot using observed absorbance data of branded and unbranded sesame oil samples. In the two-dimensional graph of score plot (Fig.4 (a)) could potentially explained oil samples of first principal components (F1) are $97.12 \%$ variance, while the second principal components explained (F2) $2.43 \%$ variance, and, Fig. 4(b) explained oil samples of first principal components (F1) are $97.12 \%$ variance, while the third principal components explained (F3) $0.41 \%$ variance. The results indicate in the PCA of the first three principal components could possibly explain approximately $99.55 \%$ of all sample information. The displayed biplot (Fig.4 (c) and (d)) can be seen that unbranded 2 and brand 1 sesame oils is an outlier in the upper right quadrant, followed by other on the right lower quadrant. Further, each oil samples occupy different positions in the principal components 1 and 2, these results concluded that PCA allows qualitative discrimination between the branded and unbranded sesame oil samples based on this analysis. Moreover, the principal component analyses of FTIR spectra indicated that there was no overlapping between unbranded 2 , brand 1 and other branded oils, however other branded oils are highly overlapping each other. Therefore, chemically and structurally similar components were observed in the various branded (2-9) and unbranded (1) sesame oils used in this PCA score plot analysis. While, in unbranded 2 and brand 1 oils composed of some important principal components compared to other oil samples. This detail supported that using ATR-FTIR technique fingerprint region shall differentiate the oil samples in terms of the number of peak and the peak intensities at the finger print regions.

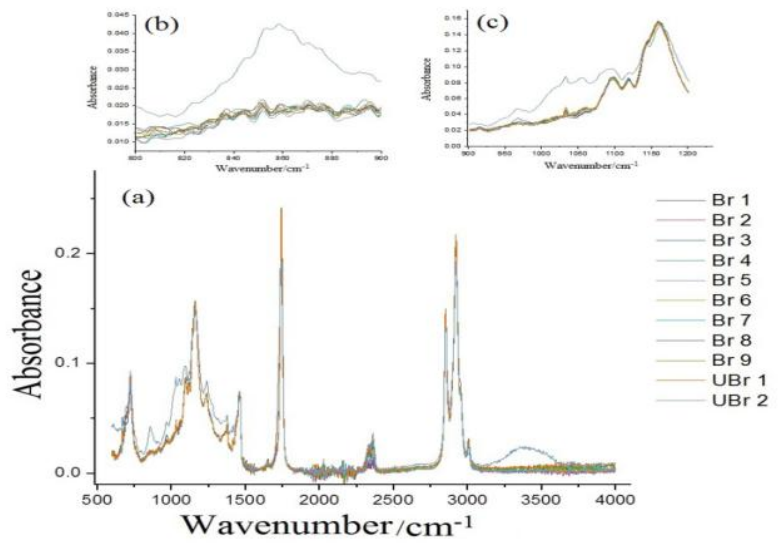

Fig. 3: FTIR absorption spectra branded (1-9) and unbranded $(1,2)$ sesame oil samples between 599-4000 cm-1 (a). Inset Figure: range between $800-900$ cm-1 (b), range between 900-1200 cm-1 (c).

\section{Agglomerative hierarchical clustering (AHC)}

In the agglomerative hierarchical approach, we define each data point as a cluster and combine existing clusters at each step. The AHC was carried out using Euclidean distance and Ward's method as the distance measure and complete linkage approach to link clusters inside the data set. Fig. 5 shows the dendrogram of the unbranded and branded sesame oil samples spectral data. Dendrogram shows that the unbranded 2 and brand 1 sesame oil samples formed one cluster, unbranded1, brand 5 and brand 2 formed second cluster and branded 3,4,6,7, 8, and 9 sesame oils formed third cluster. The observed results of AHC clearly indicates that unbranded 2 and branded 2 sesame oil having similar or maximum essential components, and other oils were missing/eliminated/adulterated with some of the components during refined/filtration process.
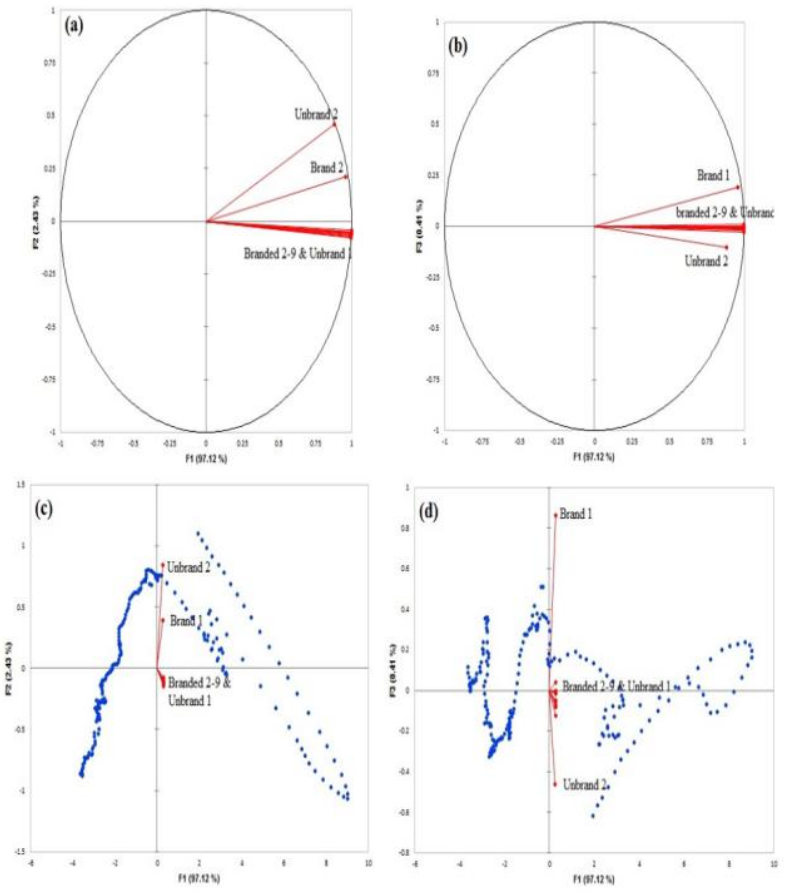

Fig. 4: Two-dimensional principal component analysis showing score plot of the first two principal components (F1 and F2) of various branded (19) and unbranded (1-2) sesame 
oil samples, variability explained F1 (97.12\%) and F2 $(2.43 \%)$ (a); score plot of first and third (F1 and F3) principal components of various branded (1-9) and unbranded (1-2) sesame oil samples, variability explained F1 $(97.12 \%)$ and F3 (0.41\%); Biplot of the first two principal components (F1 and F2) of various branded (1-9) and unbranded (1-2) sesame oil samples (c); Biplot of first and third (F1 and F3) principal components of various branded (1-9) and unbranded (1-2) sesame oil samples.

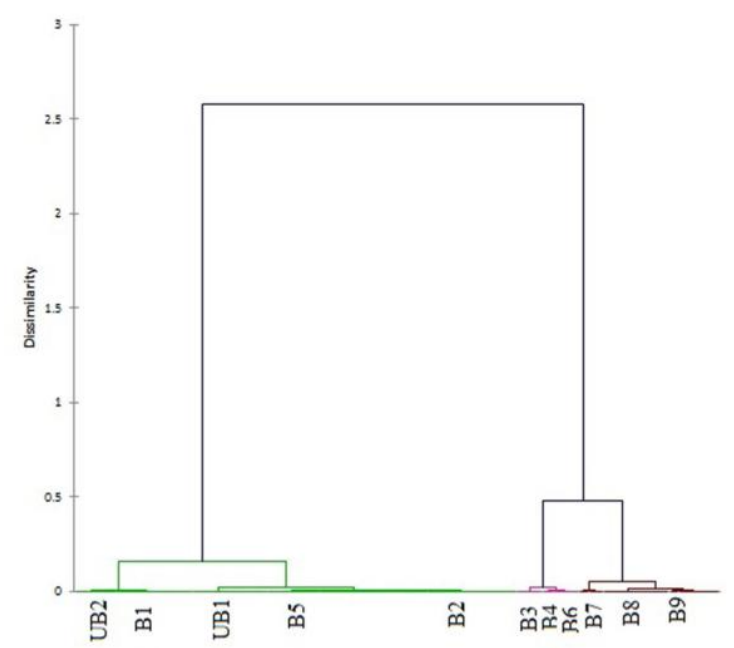

Fig. 5: Dendrogram using agglomerative hierarchical clustering of various branded (1-9) and unbranded (1-2) sesame oil samples based on the variables represented by the first two principal components.

\section{E. Classification}

Branded and unbranded sesame oil samples were classified using discriminant analysis (DA) at the frequency regions of $599-1200 \mathrm{~cm}^{-1}$. In the DA calibration model, the determination of the linearity of the techniques was calibrated in this way to show a proportional relationship between predictor variables (band intensity) and the percentage of principal components present in the branded and unbranded sesame oil samples. Fig. 6 (a) display the correlation between different oil samples against various axes of principal components analyses using DA. Fig. 6(b) the similarity map as established by discriminant factors $\mathrm{c} 1$ and $c 2$, which explains total variance.
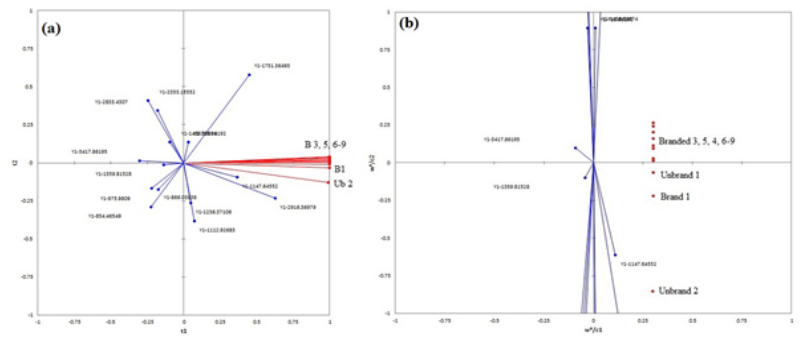

Fig. 6: The correlation between various branded (1-9) and unbranded (1-2) sesame oil samples against various axes of principal components (a); similarity map as defined by discriminant factors $\mathrm{c} 1$ and $\mathrm{c} 2$ (b).

\section{F. Principal Coordinate analysis (PCoA)}

Figure 7 explore and visualized the similarities of branded and unbranded sesame oil samples based on PCoA, it is projecting the distances in to Euclidean space for grouping. The unweighted pair-group centroid strategy, which characterizes the distance between two groups as the distance between their centroids (center of gravity or vector average), was used to compare these groups and to draw conclusions about their differences. Unbranded oil 2 is a group 1, branded oil 1 is a group 2 and other oils samples were group 3. The distance between groups 1 and 2 was 79.66, between groups 2 and 3 was 19.91, and between groups 1 and 3 was 99.48 .

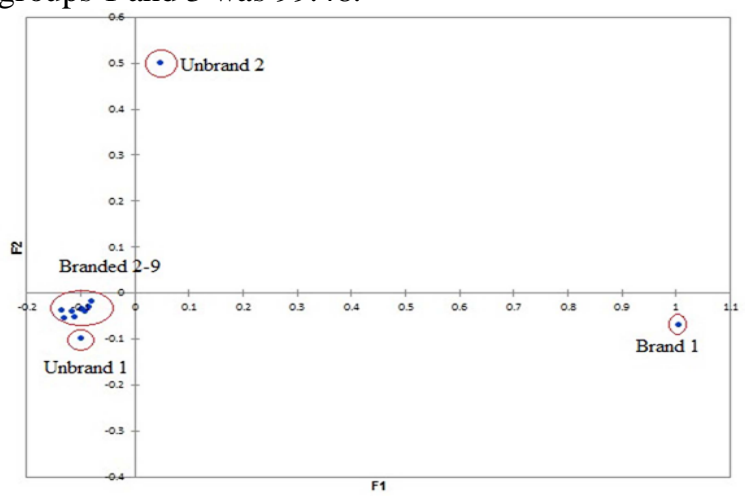

Fig. 7: The similarities of branded and unbranded sesame oil samples using principal coordinate analysis.

\section{CONCLUSION}

The combination of ATR-FTIR associated with multivariate chemometric techniques can effectively detect and identify the essential principal components presence in the various branded and unbranded edible sesame oil samples. This approach of PCA, PCoA, AHC and DA can assist the classification and quantification of principal components in branded and unbranded edible sesame oils used in food quality measurement. The selected technique was simple, rapid, and non-destructive with a total analysis time less than 5 min for each measurement. Furthermore, it is environmental friendly and avoids time consuming experimental process, toxic chemicals and solvents can be evaded.

\section{REFERENCES}

1. X Wei, H Gong, J Yu, et al., SesameFG, an integrated database for the functional genomics of sesame. Scientific Reports 2017, 7, 2342.

2. P Bigoniya, R Nishad and C S Singh, Preventive effect of sesame seed cake on hyperglycemia and obesity against high fructose-diet induced Type 2 diabetes in rats. Food Chem, 2012, 133, 1355-1361.

3. J Murata, E Ono, S Yoroizuka, et al., Oxidative rearrangement of (+)-sesamin by CYP92B14 co-generates twin dietary lignans in sesame. Nature Commun, 2017, 8, 2155.

4. N Ramarathnam, T Osawa, H Ochi, et al., The contribution of plant food antioxidants to human health. Tr Food Sci Technol, 1995, 6, 75-82.

5. A T Petkoska and A T Broach, Antioxidant compositions and methods of protecting skin, hair and nails against high energy blue-violet light. Google Patents, 2016.

6. N Vlachos, Y Skopelitis, M Psaroudaki, et al., Applications of Fourier transform-infrared spectroscopy to edible oils. Anal Chim Acta, 2006, 573, 459-465.

7. A P Simopoulos. The importance of the ratio of omega6/omega-3 essential fatty acids. Biomed Pharmacotherapy, 2002, 56, 365-379.

8. $\mathrm{T}$ Ulbricht and D Southgate, Coronary heart disease, seven dietary factors. The Lancet 1991, 338, 985-992.

9. R P Mensink and M B Katan, Effect of dietary fatty acids on serum lipids and lipoproteins. A metaanalysis of 27 trials. 
Arteriosclerosis and thrombosis, a J Vascular Biol, 1992, 12, 911-919.

10. N Shah, Sesamum indicum (Sesame or Til), Seeds and Oil-A Historical and Scientific Evaluation from Indian Perspective. Asian Agri-History, 2016, 20.

11. M Elleuch, S Besbes, O Roiseux, et al., Quality characteristics of sesame seeds and by-products. Food Chem, 2007, 103, 641650.

12. E Betiku and T F Adepoju, Methanolysis optimization of sesame (Sesamum indicum) oil to biodiesel and fuel quality characterization. Int J Energy Environ Eng, 2013, 4, 9.

13. H Yang, J Irudayaraj and M M Paradkar, Discriminant analysis of edible oils and fats by FTIR, FT-NIR and FT-Raman spectroscopy. Food Chem, 2005, 93, 25-32.

14. A Rohman and Y C Man, Fourier transform infrared (FTIR) spectroscopy for analysis of extra virgin olive oil adulterated with palm oil. Food Res Int, 2010, 43, 886-892.

15. Y W Lai, E K Kemsley and R H Wilson, Potential of Fourier transform infrared spectroscopy for the authentication of vegetable oils. J Agri Food Chem, 1994, 42, 1154-1159.

16. L Rodriguez-Saona and M Allendorf, Use of FTIR for rapid authentication and detection of adulteration of food. Ann Rev Food Sci Technol, 2011, 2, 467-483.

17. L M Reid, C P O'donnell and G Downey, Recent technological advances for the determination of food authenticity. Tr Food Scie Technol, 2006, 17, 344-353.

18. AOAC, 1993, Official Methods and Best Practices of the American, Oil Chemists Society. $3^{\text {rd }}$ edition, Champaign, Illinois.

19. ISO 3657, 2013. Animal and vegetable fats and oils determination of saponification value. Edition 4, 10. http,//www.iso.org/standard/60526.html.

20. M E Tipping and C M Bishop, Probabilistic principal component analysis. J Royal Statistical Soc, Series B (Statistical Methodology), 1999, 61, 611-622.

21. E Martin and A Morris, Non-parametric confidence bounds for process performance monitoring charts. J Process Control, 1996, 6, 349-358

\section{AUTHOR PROFILE}

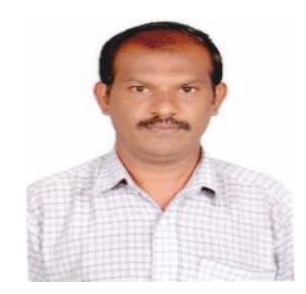

K. Selvaraj received B.Pharm. from Tamilnadu Dr. MGR Medical University in 1999, and Ph.D. from Jadavpur University., Kolkata in 2015 . He is presently working as an Assistant Professor in Department of Biotechnology, Kalasalingam University, Tamilnad. He has won Erasmus Mundus Fellowship, University of Warsaw, Poland.

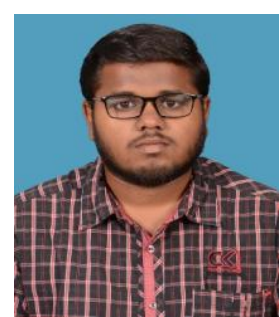

B. Suraj received B.Tech., in 2016 and M.Tech., in 2019 from Kalasalingam University, Tamilnadu, India. Currently working on nano-particle formulation and targeted drug delivery for cancer systems.
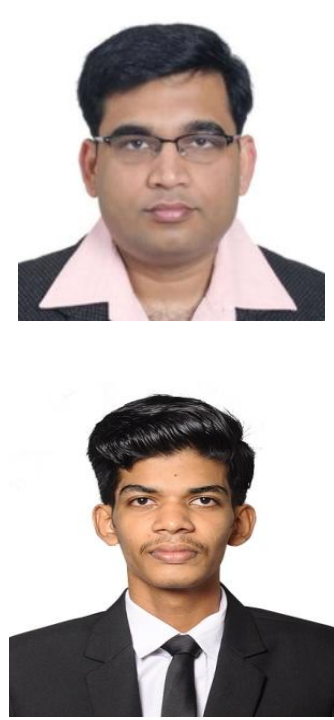

P Sajan Francis doing bachelor of pharmacy at Karavali college of pharmacy, NH 169, vamanjoor, Mangalore-575028.

A.Sankar Ganesh received B.Sc., in 1998 from Madras University and M. Sc., in 2001 from Madras University. $\mathrm{He}$ is presently working in Kalasalingam Academy of Research and Education. 